\title{
Percepção dos consumidores sobre a aplicação de revestimentos comestíveis em carne
}

\section{bovina fresca}

\author{
Consumer perceptions of the application of edible coatings on fresh beef \\ Percepciones de los consumidores sobre la aplicación de recubrimientos comestibles en la carne
} vacuna fresca

Recebido: 27/01/2022 | Revisado: 05/02/2022 | Aceito: 11/02/2022 | Publicado: 16/02/2022

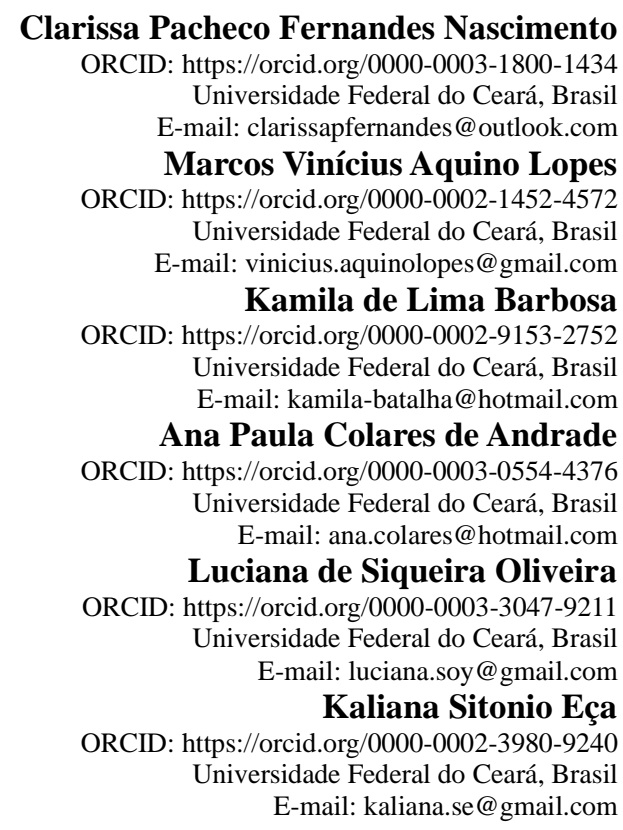

\begin{abstract}
Resumo
Esta pesquisa teve como objetivo fazer um levantamento sobre a percepção dos consumidores na utilização de revestimentos comestíveis (RC) em carne bovina fresca, como tecnologia de preservação, e sua influência na aquisição e nos hábitos de consumo desse produto. A coleta de dados ocorreu por meio de questionário online, divulgado através de mídias sociais no território nacional e respondido voluntariamente. Foram avaliadas 423 respostas, sendo em sua maioria do gênero feminino (72,8\%), com idade de 18 a 35 anos (70,9\%), solteiro (61,9\%), com alta escolaridade $(87,8 \%)$ e renda familiar mensal de dois a quatro salários mínimos $(25,1 \%)$. Observou-se que a maioria dos participantes que realizam a compra de alimentos para suas casas $(61,2 \%)$, tem preferência de fazê-la em supermercados $(62,2 \%)$, consideram o odor e a aparência da carne bovina os atributos mais importantes para a sua aquisição $(64,8 \%$ e $64,4 \%$, respectivamente) e consomem esse alimento de duas a quatro vezes por semana (47,8\%). Os participantes têm familiaridade com o tema de RC (34,7\%), consideram sua utilização muito importante para os alimentos e que a informação sobre o RC esteja presente no rótulo (96\%). Sobre a aplicação do RC na carne bovina, os participantes consideram que essa tecnologia é muito interessante para a conservação desse alimento (51,1\%), porém têm curiosidade em conhecer o produto para obterem as próprias conclusões (73,2\%). Diante do exposto, concluímos que os consumidores acreditam que a aplicação de RC pode ter um grande potencial para conservação da carne bovina fresca durante o armazenamento.

Palavras-chave: Comportamento do consumidor; Produtos cárneos; Tecnologia emergente; Embalagens ativas; Segurança de alimentos.
\end{abstract}

\begin{abstract}
This study aimed to evaluate the consumers' perception about using of edible coating (EC) in fresh beef as a preservation technology and the influence on the purchase and consumption habits of this product. An online questionnaire was used, which was posted on social medial in the national territory and answered voluntarily. 423 responses were evaluated, mostly female (72.8\%), aged between 18 and 35 years (70.9\%), single (61.9\%), high education levels $(87.8 \%)$ and monthly family income from two to four minimum wages $(25.1 \%)$. Most respondents purchase food for their homes $(61.2 \%)$, prefer to do in supermarkets $(62.2 \%)$, consider the odor and appearance of
\end{abstract}


fresh beef the most important attributes for their acquisition (64.8\% and $64.4 \%$, respectively) and consume the fresh beef two to four times a week (47.8\%). The respondents are familiar with EC (34.7\%), consider that technology very important for foods and that information about EC is present on the label (96\%). About the application of EC in beef, the participants consider this practice to be very interesting for the conservation $(51.1 \%)$, but they are curious to know the product to have your own opinion (73.2\%). Thus, we conclude that consumers believe that the application of EC may have a great potential for preserving fresh beef.

Keywords: Consumer behavior; Meat products; Emerging Technology; Active packaging; Food safety.

\section{Resumen}

Este estudio tuvo como objetivo evaluar la percepción de los consumidores sobre el uso de recubrimiento comestible (EC) en carne vacuna fresca como tecnología de conservación y la influencia en los hábitos de compra y consumo de este producto. Se utilizó un cuestionario en línea, el cual fue colgado en redes sociales en el territorio nacional y respondido voluntariamente. Se evaluaron 423 respuestas, en su mayoría mujeres $(72,8 \%)$, edad entre 18 y 35 años $(70,9 \%)$, soltera $(61,9 \%)$, nivel educativo alto $(87,8 \%)$ y renta familiar mensual de dos a cuatro salarios mínimos $(25,1 \%)$. La mayoría de los encuestados compran alimentos para sus hogares $(61,2 \%)$, prefieren hacerlo en los supermercados $(62,2 \%)$, consideran el olor y la apariencia de la carne vacuna fresca los atributos más importantes para su adquisición $(64,8 \%$ y $64,4 \%$, respectivamente) y consumen la carne fresca. Carne de res de dos a cuatro veces por semana $(47,8 \%)$. Los encuestados están familiarizados con la CE (34,7\%), consideran que la tecnología es muy importante para los alimentos y que la información sobre la CE está presente en la etiqueta (96\%). Sobre la aplicación de la CE en la carne vacuna, los participantes consideran esta práctica muy interesante para la conservación $(51,1 \%)$, pero tienen curiosidad por conocer el producto para tener una opinión propia $(73,2 \%)$. Por lo tanto, concluimos que los consumidores creen que la aplicación de la CE puede tener un gran potencial para conservar la carne vacuna fresca.

Palabras clave: Comportamiento del consumidor; Productos de carne; Tecnología emergente; Envases activos; Seguridad alimenticia.

\section{Introdução}

O mercado global de alimentos tem sido motivado em acompanhar a crescente demanda de consumidores mais exigentes aos critérios de qualidade, segurança, naturalidade e preocupações ambientais (Khedri et al., 2021). Uma tecnologia apropriada para atender essa demanda é a utilização de revestimentos comestíveis formulados com biopolímeros, visando prolongar a estabilidade de produtos perecíveis ao longo do armazenamento (Mohamed et al., 2020). Esses revestimentos são aplicados sobre a superfície do alimento e atuam como uma barreira semipermeável que reduz a troca de umidade, de gases e compostos voláteis, além de poder ser utilizado como transportadores de compostos funcionais naturais (Cazón et al., 2017), como antioxidantes, antimicrobianos, aromatizantes e corantes (Falguera et al., 2011; Sahraee et al., 2019; Zhang et al., 2018).

A carne bovina é um importante alimento da dieta humana, sendo fonte de proteínas, vitaminas, minerais e micronutrientes (Umaraw et al., 2020). Entretanto, é um alimento suscetível à deterioração, que pode ocorrer devido à oxidação de lipídios e proteínas e deterioração microbiana, representando um alto risco para a saúde do consumidor, além das perdas econômicas para o mercado (Nikmaram et al., 2018). De acordo com estudos, o uso dos revestimentos comestíveis em carnes é considerado uma alternativa para minimizar essas alterações relacionadas aos aspectos de segurança e qualidade (Bermúdez-Oria et al., 2019; Trejo-González et al., 2018).

A manutenção dos aspectos intrínsecos e extrínsecos da carne, além de ser uma preocupação para a indústria, é de extrema importância para a aceitação pelo consumidor (Maurício, 2020). Diversos trabalhos (Behbahani et al., 2020; Utami et al., 2016; Vital et al., 2018) investigam os efeitos da aplicação dos revestimentos comestíveis na qualidade da carne, porém há poucos dados sobre o comportamento do consumidor em relação à esta tecnologia.

Dessa forma, o presente trabalho teve como objetivo compreender a percepção dos consumidores em relação à tecnologia de revestimentos comestíveis e sua aplicação em carne bovina, utilizando um questionário online, além de verificar o perfil do mercado de aquisição e consumo desse alimento. 


\section{Metodologia}

A pesquisa foi realizada através de um estudo de cunho quantitativo e caráter descritivo utilizando um questionário criado na plataforma Google, previamente elaborado pelos pesquisadores e aprovado pelo Comitê de Ética em Pesquisa (CEP) da Universidade Federal do Ceará sob parecer $\mathrm{n}^{\circ}$ 4.912.426. O questionário foi dividido em três partes principais: 1) perfil sociodemográfico, 2) perfil de aquisição e consumo de carne bovina e 3) percepções sobre os revestimentos comestíveis e sua aplicação na carne bovina (Quadro 1).

Quadro 1 - Perguntas realizadas por meio do Formulário online da Plataforma Google.

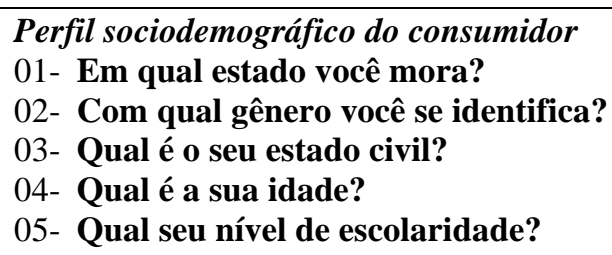
listados são importantes para você na aquisição da carne bovina: __Origem/Procedência __Aparência __ Quantidade de gordura __Odor __Embalagem Preço

Percepções sobre os revestimentos comestiveis $\boldsymbol{e}$ sua aplicação na carne bovina

10- Você já estava familiarizado com a tecnologia dos revestimentos comestíveis para alimentos?

11- Para você, quão importante é o uso dos revestimentos comestíveis?

12- Caso o alimento tenha sido revestido, para você, é importante que essa informação esteja presente no rótulo?

13- Na escala de 1 (não é importante) a 5 (muito importante), indique o quanto você considera que a aplicação de revestimentos comestíveis pode ser interessante na conservação de carnes bovina.

14- De acordo com a imagem abaixo, indique em qual dos lados foi a aplicação de revestimentos

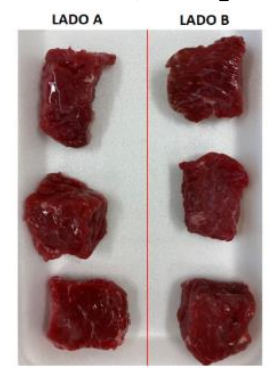
comestíveis nas carnes:

15- Você está disposto(a) a comprar/consumir esse produto? (carne bovina revestido por materiais comestíveis e biodegradáveis).

Fonte: Autores (2021).

Os participantes foram convidados a responderem o questionário de forma remota através de mídias sociais (WhatsApp, Facebook, Instagram e LinkdIn) no território nacional, Brasil, durante os meses de julho e agosto de 2021, demonstrando interesse de participação através da aceitação do Termo de Consentimento Livre e Esclarecido (TCLE).

O tamanho amostral foi calculado por meio de site especializado (Survey Monkey) considerando a população atual do Brasil (212,6 milhões), em intervalo de confiança de 95\% e 5\% de erro amostral. Para análise estatística, os dados foram 
armazenados e tabulados na ferramenta Microsoft Excel® e para determinados dados qualitativos o teste qui-quadrado foi aplicado $(\mathrm{p}<0,05)$ com ajuda do programa Jamovi versão 1.6.23.

\section{Resultados e Discussão}

Foram obtidas 423 respostas, observando à abrangência do questionário em todas as regiões do Brasil. A maioria das participações foi obtido da região Nordeste, nos estados do Ceará, Rio Grande do Norte e Maranhão (185, 143 e 26 respostas, respectivamente), seguida da região Sudeste, no estado de São Paulo (20 respostas). Em contrapartida, a minoria das participações foi nos estados do Pará, Santa Catarina, Rio Grande do Sul e Sergipe (uma resposta em cada estado) (Figura 1).

Essa distribuição territorial faz-se interessante nesta pesquisa, uma vez que se trata de um estudo sobre a intenção de compra de carne bovina com revestimentos comestíveis, abordando a caracterização sociodemográfica dos entrevistados, a coleta de informações sobre seus hábitos de compra, frequência de consumo de carne bovina e os fatores que afetam essas práticas, além de explorar o nível de entendimento dos indivíduos sobre o tema abordado no questionário.

Figura 1 - Frequência de respostas obtidas nos estados brasileiros.

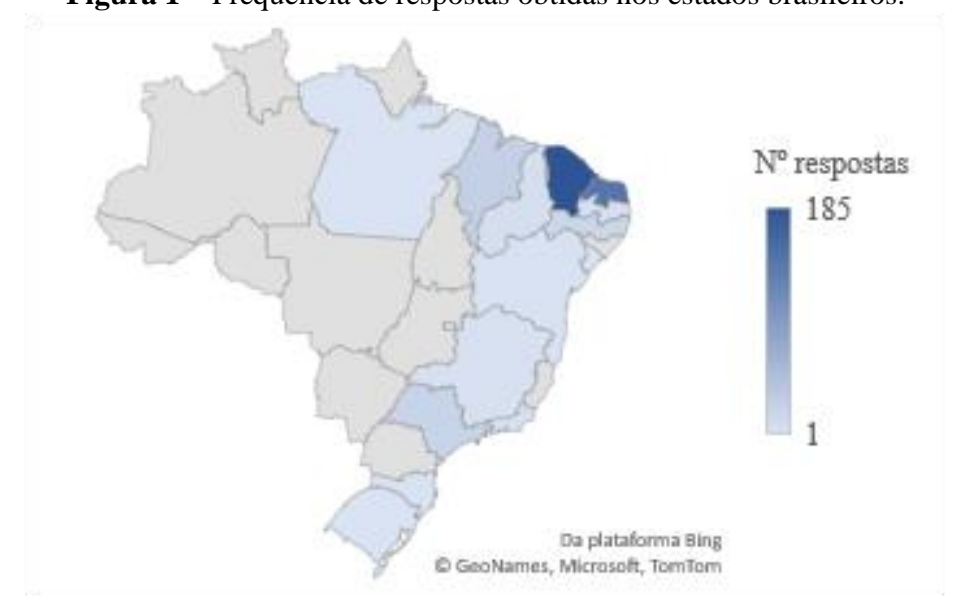

Fonte: Autores (2021).

De acordo com os dados levantados, a amostra foi composta, em sua maioria, por pessoas do gênero feminino $(72,8 \%)$ (Tabela 1). Semelhança encontrada por outros autores e que reflete o papel atribuído às mulheres como as principais responsáveis pela aquisição de alimentos para as suas famílias (Frabasile, 2016; Schaly et al., 2010). Quanto ao estado civil, os solteiros corresponderam a 61,9\% (Tabela 1). Esse perfil é contrário com os encontrados em outros levantamentos sobre o perfil do consumidor da carne bovina, onde verificaram um maior percentual de entrevistados casados em relação a outros estados civis (Borges, Nunes \& Cavalcante, 2020; Dias et al., 2015; Nascimento et al., 2018).

Observou-se a participação de indivíduos de várias idades (de 18 à parcela acima de 65 anos), sendo a faixa de 18 a 35 anos com a maior participação, somando 70,9\% (Tabela 1). O predomínio dessas idades também foi notado por outros autores (Brandão et al., 2012; Dias et al., 2015) e se trata de um público adulto jovem, nomeado Geração Z, que emerge como alvo de ações mercadológicas por se tratar de um público com autonomia de escolha, exigente e com potencial de influência nas decisões de compra da família (Ceretta \& Froemming, 2011; Coray, 2019).

Com relação ao grau de instrução, a maioria dos indivíduos tinha, pelo menos, ingressado no ensino superior (29,1\% ensino superior incompleto, $19,9 \%$ superior completo, 10,2\% pós-graduação incompleta e $28,6 \%$ pós-graduação completa), o que configura um público que tem acesso à informação e com certo nível de discernimento para responder o questionário proposto (Tabela 1). Apenas 1,6\% deles declararam não possuírem os ensinos fundamental ou médio completos (Tabela 1), 
porcentagem bastante inferior ao censo do Instituto Brasileiro de Geografia e Estatística (IBGE) em 2019 (IBGE, 2019). Essas observações são compatíveis aos encontrados em outros estudos abordando a carne bovina, onde mais da metade dos entrevistados apresentavam alta escolaridade (Brandão et al., 2012; Schaly et al., 2010).

O nível de escolaridade é uma variável de grande influência na escolha e exigência de produtos cárneos de modo que, o maior grau de escolaridade por parte dos consumidores, como visto neste trabalho, favorece a conscientização dos mesmos em exigir carnes de melhor qualidade e padrão sanitário (Borges et al., 2020; Kirinus et al.,2013).

Com relação à renda familiar dos entrevistados, observou-se uma representação variada desse perfil. Considerando o valor do salário mínimo (SM) em 2021 de $\mathrm{R} \$ 1100,00$, a classe que ganha de dois a quatro SM foi majoritária nessa pesquisa (25\%), seguido das rendas de até dois SM (20,3\%) e até um SM (18,2\%). As demais faixas salariais, de quatro a seis SM, seis a dez SM e acima de dez SM apresentarem percentuais de 15,8, 11,1 e 9,5\%, respectivamente (Tabela 1). Estes resultados apontam que $63,6 \%$ dos entrevistados apresentam renda de até quatro SM, sendo compatível com as rendas salariais encontradas nas pesquisas de Dias et al. (2015), Nascimento et al. (2018) e Schaly et al. (2010). O nível de renda é um fator crucial para determinar o consumo familiar de carne, pois seu aumento eleva as chances de despesas com esse tipo de alimento (Schlindwein \& Kassouf, 2006).

Tabela 1 - Distribuição dos indivíduos segundo os dados sociodemográficos.

\begin{tabular}{lll}
\hline Variável & Categoria de respostas & $\begin{array}{l}\text { Frequência (\%) } \\
(\mathbf{n}=\mathbf{4 2 3})\end{array}$ \\
\hline Gênero & Feminino & 72,8 \\
Faixa etária & Masculino & 27,2 \\
& $18-25$ & 32,6 \\
& $26-35$ & 38,3 \\
& $36-45$ & 12,3 \\
Estado civil & $46-55$ & 6,1 \\
& 56-55 & 6,9 \\
& Acima de 65 & 3,8 \\
& Solteiro(a) & 61,9 \\
Grau de & Casado(a)/União estável & 35,5 \\
escolaridade & Divorciado(a) & 1,9 \\
& Viúvo(a) & 0,7 \\
& Fundamental incompleto & 0,5 \\
& Fundamental completo & 0,2 \\
& Médio incompleto & 0,9 \\
& Médio completo & 10,6 \\
& Superior incompleto & 29,1 \\
& Superior completo & 19,9 \\
Renda familiar & Pós-graduação incompleta & 10,2 \\
& Pós-graduação completa & 28,6 \\
& & \\
& Até 1 SM & 18,2 \\
& Até 2 SM & 20,3 \\
& De 2 a 4 SM & 25,1 \\
& De 4 a 6 SM & 15,8 \\
& De 6 a 10 SM & 11,1 \\
& Acima de 10 SM & 9,5 \\
\hline
\end{tabular}

SM: salário mínimo. Fonte: Autores (2021).

Em relação ao perfil de compra e consumo de carne bovina, inicialmente ao realizar a pergunta "Quem geralmente faz as compras de produtos alimentícios na sua casa?” (Quadro 1), 61,2\% afirmaram que são os principais responsáveis por essa atividade. Esse dado é de grande relevância para o desenvolvimento desta pesquisa, visto que se trata de um público que tem 
conhecimento dos alimentos que estão disponíveis nos mercados e com capacidade de discernir sobre seus atributos de qualidade.

Considerando esse público, avaliou-se a preferência quanto aos estabelecimentos para aquisição da carne bovina e, desta forma, evidenciou-se uma tendência em concentrá-la em um só lugar, visto que $62,2 \%$ dos entrevistados responderam que adquirem a carne em supermercados, seguido por açougue/frigorífico com 31,8\%. Feira livre/mercado público ou o próprio produtor também foram mencionados com 5,5\% e 0,6\%, respectivamente (Figura 2). Amaral et al. (2012), Magalhães et al. (2021) e Eiras et al. (2017) também relataram em seus estudos a preferência pelos consumidores em adquirir a carne fresca em supermercados.

Figura 2 - Frequência de respostas da preferência de estabelecimentos para aquisição de carne bovina de pessoas que realizam as compras de produtos alimentícios de casa.

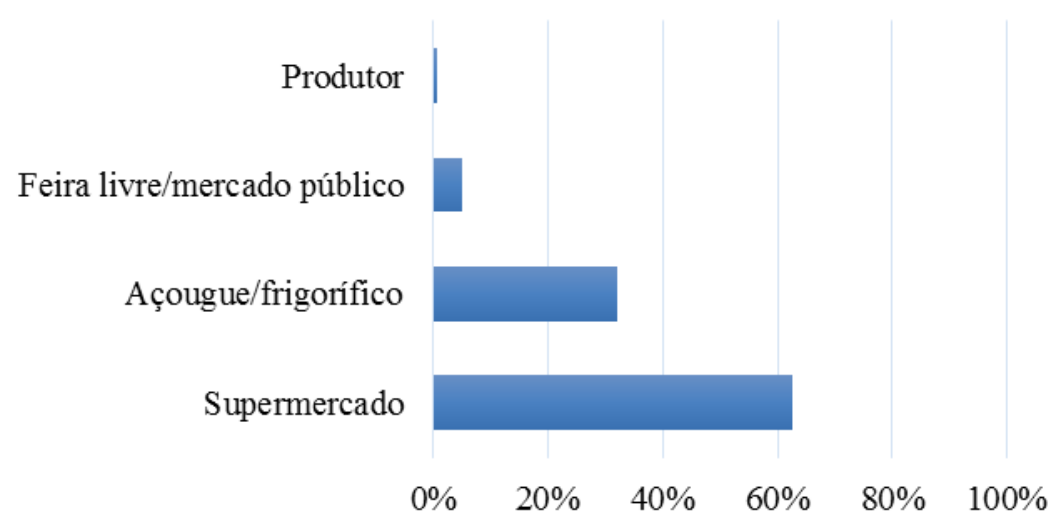

Fonte: Autores (2021).

Esse comportamento pode estar relacionado à redução do tempo disponível da população, em virtude do estilo de vida moderno. O supermercado auxilia as pessoas na aquisição de diversos tipos de produtos em um mesmo local. Além disso, dáse a esse estabelecimento uma relação de local limpo e higiênico (Eiras et al., 2017; Nascimento et al., 2018). No caso da compra em açougues/frigorífico, essa prática também está vinculada à comodidade e praticidade oferecida por esses pontos de venda, como a proximidade da residência e fidelidade ao estabelecimento (Brisola \& Castro, 2005).

O grau de importância de atributos de qualidade, econômico e de segurança que influenciam na decisão de aquisição da carne bovina também foi associado com a porcentagem de indivíduos que realizam a compra de produtos alimentícios de suas casas. "Odor" e "aparência (cor, brilho e textura)" receberam as maiores frequências de respostas como "muito importante". Do total de entrevistados, $64,8 \%$ e $64,4 \%$ rotularam essas características, respectivamente, como as mais relevantes, seguido por "origem/procedência" com 54,2\%. Verificou-se também que o "preço" foi o quarto atributo considerado mais importante (47,4\%) e "embalagem" o quinto atributo (40,3\%). As pessoas se manifestaram menos exigentes quanto à quantidade de gordura (muito importante $=28,5 \%$ ) (Figura 3 ). 
Figura 3 - Frequência de respostas do grau de importância dos atributos na aquisição de carne bovina pelos indivíduos responsáveis pela compra de produtos alimentícios.

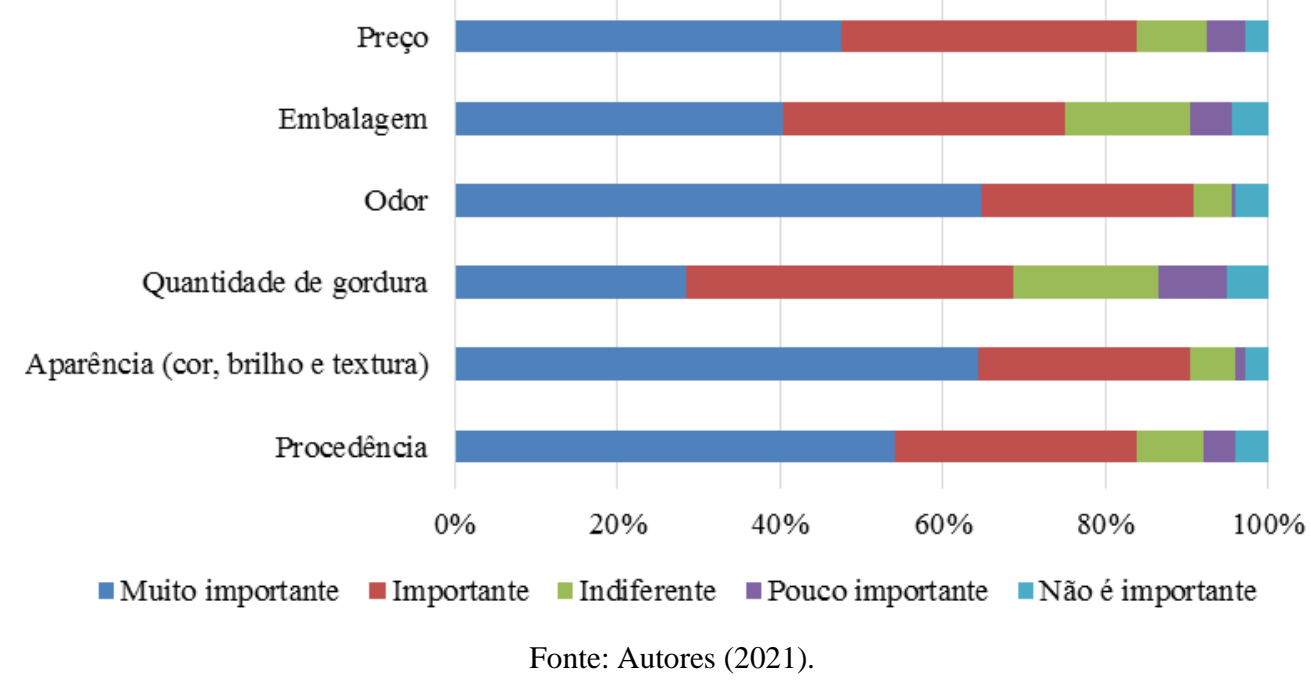

Ao analisar uma carne, seus aspectos intrínsecos (odor e aparência) desempenham um papel fundamental na aceitação desse produto como indicadores sugestivos de segurança e qualidade (Borges et al., 2020). Tal fato explica os resultados desta pesquisa, uma vez que os aspectos sensoriais apresentaram grande importância. Além disso, o valor a ser pago pela carne pode estar agregado a esses aspectos, já que, para alguns consumidores tal associação está intimamente relacionada, ou seja, o que tem mais qualidade é mais caro (Lopes et al., 2017; Maurício, 2020).

Em relação à frequência de consumo de carne bovina, percebeu-se que a maioria dos entrevistados consome a carne de 2 a 4 vezes por semana (47,8\%), seguido daqueles que consomem de 1 a 3 vezes/mês, representando 21,3\% (Figura 4). Dias et al. (2015) observou que 45,92\% dos consumidores entrevistados de Campo Grande (MS) comem carne diariamente. Assim como Cipriano et al. (2021), que verificaram um consumo de pelo menos uma vez na semana dos entrevistados do estado de Roraima.

Figura 4 - Frequência de respostas segundo o consumo de carne bovina.

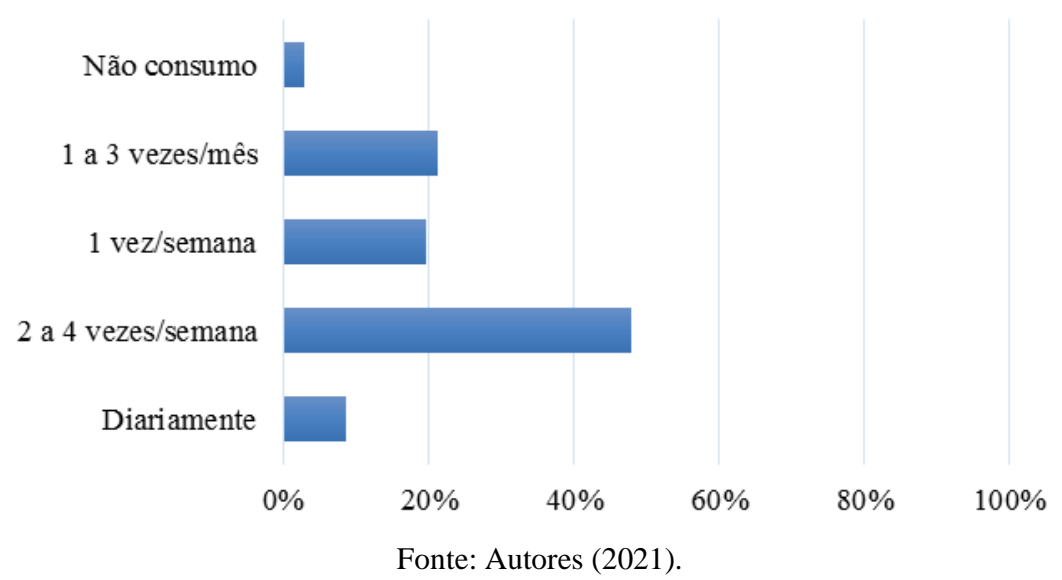

Correlacionando o perfil sociodemográfico dos entrevistados com as maiores porcentagens de consumo visualizadas na Figura 4 (de 2 a 4 vezes/semana e 1 a 3 vezes/mês), não se observou alguma correlação (p>0,05) entre os parâmetros de 
perfil através do teste qui-quadrado (Tabela 2). Esse fato revela um consumo frequente dos indivíduos e a preferência por esse alimento independente das condições sociodemográficas.

De acordo com Albuquerque et al. (2017), a carne bovina é um dos alimentos mais desejados pelos brasileiros, pela imagem social que ela transmite, isto é, a necessidade de mostrar certa ascensão social, e por ser um dos alimentos que causam maior sensação de saciedade. Além disso, os consumidores adquirem carne bovina porque gostam, demonstram atitude favorável e simpática ao consumi-la, diferente de outros alimentos que são consumidos por obrigação (Albuquerque, 2017).

Tabela 2 - Perfil amostral em relação ao consumo de carne bovina de 2 a 4

vezes/semana e 1 a 3 vezes/mês dos entrevistados.

\begin{tabular}{|c|c|c|c|}
\hline \multirow{2}{*}{$\begin{array}{l}\text { Com qual frequência você } \\
\text { consome carne bovina? }\end{array}$} & \multicolumn{2}{|c|}{ Categoria de respostas } & \multirow{2}{*}{$\begin{array}{l}\text { Valor-p } \\
(n=292)\end{array}$} \\
\hline & $\begin{array}{l}2 \text { a } 4 \text { vezes/ } \\
\text { semana }(\%)\end{array}$ & $\begin{array}{l}1 \text { a } 3 \text { vezes/ } \\
\text { mês }(\%)\end{array}$ & \\
\hline \multicolumn{4}{|l|}{ Gênero } \\
\hline Feminino & 52,4 & 22,3 & \multirow{2}{*}{0,523} \\
\hline Masculino & 16,8 & 8,6 & \\
\hline \multicolumn{4}{|l|}{ Faixa etária } \\
\hline $18-25$ & 21,6 & 9,2 & \multirow{6}{*}{0,277} \\
\hline $26-35$ & 27,1 & 14,7 & \\
\hline $36-45$ & 9,2 & 2,4 & \\
\hline $46-55$ & 4,5 & 1,7 & \\
\hline $56-65$ & 5,1 & 1,0 & \\
\hline Acima de 65 & 1,7 & 1,7 & \\
\hline \multicolumn{4}{|l|}{ Estado civil } \\
\hline Solteiro(a) & 40,8 & 21,6 & \multirow{4}{*}{0,588} \\
\hline Casado(a)/União estável & 26,7 & 8,9 & \\
\hline Divorciado(a) & 1,0 & 0,0 & \\
\hline Viúvo(a) & 0,3 & 0,3 & \\
\hline \multicolumn{4}{|l|}{ Escolaridade } \\
\hline Fundamental incompleto & 0,3 & 0,0 & \multirow{8}{*}{0,531} \\
\hline Fundamental completo & 0,0 & 0,0 & \\
\hline Médio incompleto & 0,3 & 0,0 & \\
\hline Médio completo & 6,8 & 2,1 & \\
\hline Superior incompleto & 18,5 & 9,2 & \\
\hline Superior completo & 14,7 & 4,1 & \\
\hline Pós-graduação incompleta & 7,9 & 4,5 & \\
\hline Pós-graduação completa & 20,5 & 11,0 & \\
\hline \multicolumn{4}{|l|}{ Renda familiar } \\
\hline Até $1 \mathrm{SM}$ & 12,3 & 7,2 & \multirow{6}{*}{0,113} \\
\hline Até $2 \mathrm{SM}$ & 11,0 & 7,2 & \\
\hline De 2 a 4 SM & 18,2 & 6,8 & \\
\hline De 4 a $6 \mathrm{SM}$ & 13,0 & 3,8 & \\
\hline De 6 a 10 SM & 6,2 & 4,1 & \\
\hline Acima de $10 \mathrm{SM}$ & 8,6 & 1,7 & \\
\hline
\end{tabular}

Teste qui-quadrado (p<0,05). SM: salário mínimo. Fonte: Autores (2021).

Referente às questões sobre as percepções dos entrevistados sobre os revestimentos comestíveis, 34,7\% responderam ter algum conhecimento sobre o tema, porém nunca consumiram alimentos que fizeram o uso dessa tecnologia. Contudo, 
45,3\% avaliaram como "muito importante" o uso dessa tecnologia na preservação dos alimentos; e, caso este seja revestido, $96 \%$ consideram importante a presença da informação no rótulo (Tabela 3).

Tabela 3 - Frequência de respostas sobre os revestimentos comestíveis.

\begin{tabular}{ll}
\hline Variável & $\begin{array}{l}\text { Frequência (\%) } \\
(\mathrm{n}=423)\end{array}$ \\
\hline $\begin{array}{l}\text { Você já estava familiarizado com a tecnologia dos } \\
\text { revestimentos comestíveis para alimentos? }\end{array}$ & \\
$\begin{array}{l}\text { Sim, inclusive já consumi alimentos com revestimentos } \\
\text { comestíveis. }\end{array}$ & 28,8 \\
Sim, mas nunca consumi alimentos com revestimentos & 34,7 \\
comestíveis. & 32,1 \\
Não, mas tenho interesse pelo assunto e em consumir. & 4,5 \\
Não tenho interesse pelo assunto nem em consumir. & \\
Quão importante é o uso dos revestimentos comestíveis na & \\
preservação dos alimentos? & 45,3 \\
Muito importante & 33,7 \\
Importante & 13,9 \\
Indiferente & 3,3 \\
Pouco importante & 3,8 \\
Não é importante & \\
É importante que a informação de revestimento comestível & \\
esteja presente no rótulo? & \\
Sim, considero importante. & 96 \\
Não é relevante. & 4,0 \\
\hline
\end{tabular}

Fonte: Autores (2021).

Esses resultados sugerem que os entrevistados são indivíduos sem grande resistência à tecnologia de revestimentos comestíveis, uma vez que buscam produtos com qualidade e benefícios de acordo com o que julgam relevantes para si. Além disso, julgam necessária a comunicação direta entre a indústria e os consumidores através do rótulo, podendo, então, influenciar positivamente a intenção de compra do produto com as informações presentes (Tokin et al., 2016).

É importante notar que, a amostra de entrevistados estudada foi constituída principalmente por adultos jovens cursando, pelo menos, o ensino superior, onde são indivíduos que possuem maior acesso à informação e conhecimento às inovações e, portanto, comportamentos menos neofóbicos à tecnologia de alimentos (Moura \& Masquio, 2014).

Quanto à opinião dos entrevistados sobre a aplicação do revestimento comestível na carne bovina, observou-se que pelo menos 50\% deles expressou essa prática como "muito interessante" para a conservação desse produto (Figura 5). Uma opinião positiva também foi demonstrada no estudo de Ellouze e Jeuge (2014), onde 53\% dos entrevistados também consideraram o revestimento comestível muito importante para a carne fresca, pontuando vantagens de maior vida útil, frescor e melhor qualidade organoléptica geral da carne. 
Figura 5 - Grau de importância da aplicação do revestimento comestível na carne bovina.

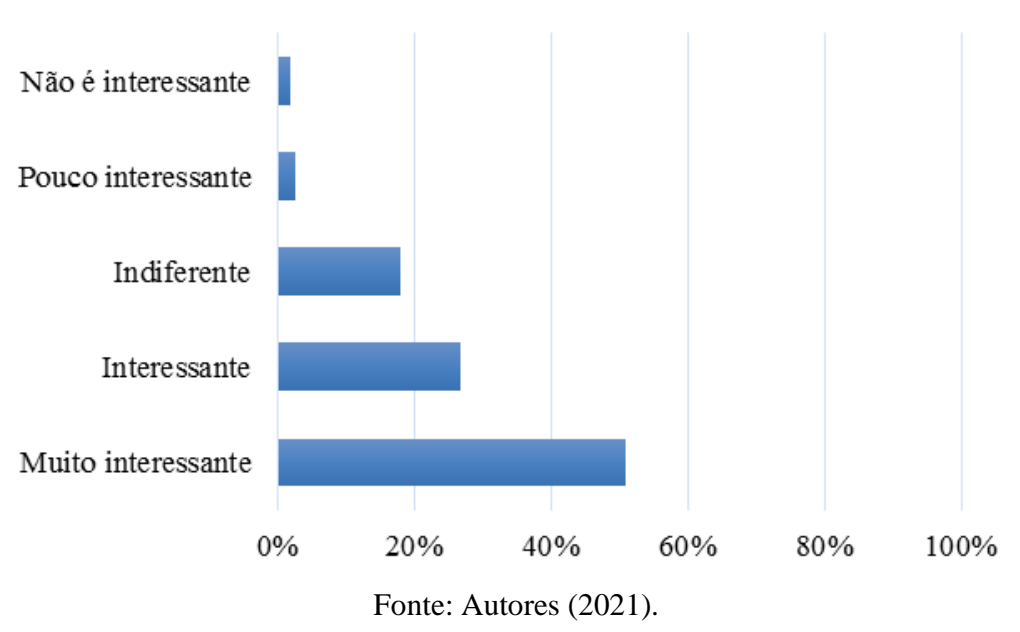

A carne fresca é um alimento de alta perecibilidade que pode sofrer deterioração durante a produção, transporte, armazenamento e consumo. A oxidação lipídica e proteica e o crescimento microbiano são as principais causas de alterações da qualidade da carne, podendo modificar sua textura, cor, sabor e reduzir a qualidade nutricional (Behbahani et al., 2020).

Nesse sentido, a aplicação de revestimentos comestíveis na superfície das carnes é uma possível alternativa para prolongar a vida útil desse produto. Esses revestimentos se comportam como uma barreira para controlar a transferência de água, gases $\left(\mathrm{O}_{2}\right.$ e $\left.\mathrm{CO}_{2}\right)$ e escape de compostos aromáticos (Bhagath \& Manjula, 2019; Souza et al., 2021). Além disso, podem incorporar aditivos naturais (extratos e óleos essenciais vegetais, probioticos etc.), como antioxidantes e antimicrobianos, que potencializam sua a funcionalidade, e, assim, minimizam os fatores acima descritos (Feng et al., 2019; Umaraw et al., 2020; Utami et al., 2016).

Em relação à figura apresentada no questionário (Quadro 1), cerca de $90 \%$ dos entrevistados conseguiram identificar a carne na qual foi aplicada o revestimento comestível. Considerando essa resposta, realizou-se uma correlação entre a identificação da figura dentre os seguintes níveis de familiaridade sobre o tema de revestimentos comestíveis: "sim, inclusive já consumi alimentos com revestimentos comestíveis", "sim, mas nunca consumi alimentos com revestimentos comestíveis" e "não, mas tenho interesse pelo assunto e em consumir". Observou-se que não houve diferença na correlação entre as imagens "lado A" e "lado B" com os níveis de conhecimento ( $\mathrm{p}>0,05)$, sendo o maior número de respostas obtido para o "lado A". Indicando a capacidade de assimilação dos revestimentos comestíveis nas imagens apresentadas entre todos os públicos estudados (Figura 6). 
Figura 6 - Frequência de respostas da identificação das imagens de carne bovina através dos níveis de familiaridade sobre o tema de revestimentos comestíveis.

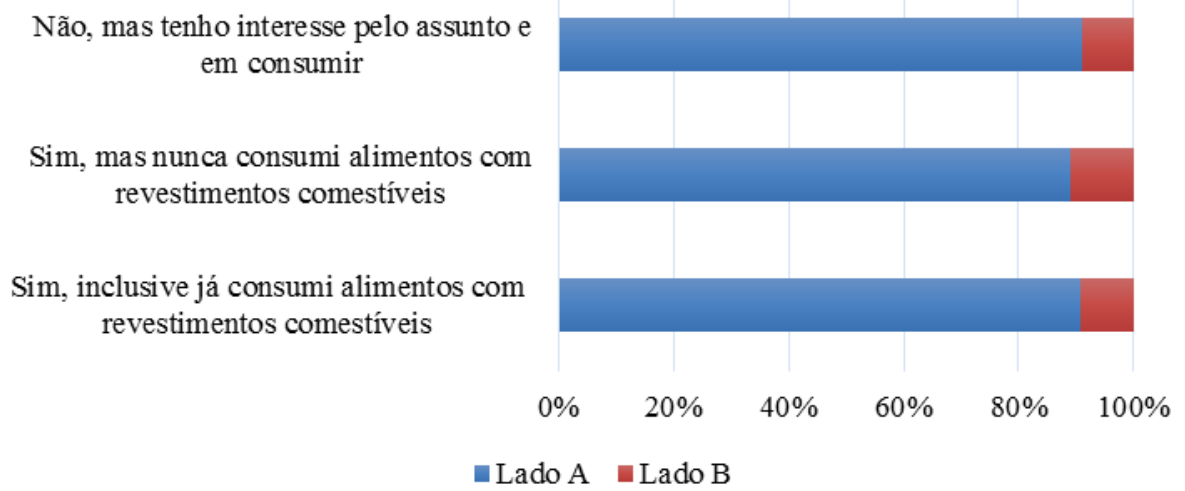

Fonte: Autores (2021).

Esta alta porcentagem de identificação das carnes revestidas pode ser devido ao seu maior brilho em comparação a controle. Esse comportamento pode ser positivo para o presente trabalho, uma vez que os consumidores acabam se baseando na perda de cor e brilho da carne como um indicativo de insalubridade e deterioração do produto (Groot, 2021; Kiarsi et al., 2020). O uso de revestimentos comestíveis tem sido uma alternativa possível para minimizar a perda dessa característica, aumentando a vida útil de carnes frescas (Cardoso et al., 2016; Umaraw et al., 2020).

Ao avaliarmos a disposição dos entrevistados a comprarem e/ou consumir a carne bovina revestida por materiais comestíveis e biodegradáveis, sendo excluídos aqueles que declararam não consumir carne, 71,9\% relataram ter curiosidade de conhecer inicialmente o produto para obter as próprias conclusões, 22\% declararam não ter problema em comprar e/ou consumir a carne e 6,1\% não estão dispostos a comprar e/ou consumi-la (Figura 7). Isso representa que os consumidores apresentam incertezas quanto ao produto, porém dispostos à experiência. Esse fator se refere às dúvidas e desconhecimento das novas aplicações tecnológicas nos alimentos objetivando o aumento da sua vida útil e segurança (Coutinho et al., 2021).

Figura 7 - Frequência de respostas da disposição de compra e/ou consumo da carne bovina com revestimentos comestíveis.

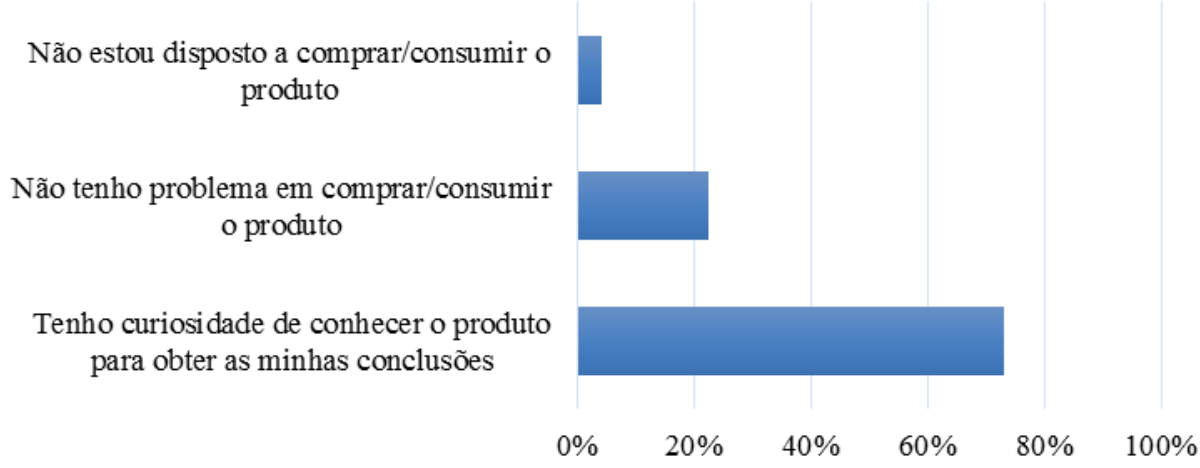

Fonte: Autores (2021).

\section{Conclusão}

Neste estudo foi possível identificar um nível satisfatório do perfil dos participantes quanto à valorização dos aspectos de qualidade da carne bovina e receptividade ao uso de revestimentos comestíveis e sua aplicação na carne bovina fresca. 
Os consumidores consideram a aplicação dos revestimentos comestíveis na carne bovina com grande potencial para a sua conservação durante o armazenamento. No entanto, eles necessitam conhecer o produto antes de qualquer coisa para que a disposição de compra seja aumentada. Isso indica que o sucesso da carne com revestimento comestível pode depender de estratégias adequadas de marketing para apresentar ao consumidor informações confiáveis, compreensíveis e claras sobre a tecnologia empregada na carne bovina fresca.

\section{Referências}

Albuquerque, I. R. R., Gois, G. C., Campos, F. S., Silva, T. S., \& Matias, A. G. S. (2017). Pesquisa de mercado: hábitos de compra e consumo de carne em Senhor do Bonfim - Bahia. Nutri-time, 14(2), 5024-5029. https://www.nutritime.com.br/arquivos_internos/artigos/Artigo_418.pdf.

Amaral, D. S., Cardoso, D. S. G., Pessoa, T., \& Moura, L. G. Neto. (2012). Perfil dos consumidores da carne de sol comercializada nos municípios de em Caicó e Currais Novos - RN. Acta Veterinaria Brasilica, 6(4), 302-311. https://periodicos.ufersa.edu.br/index.php/acta/article/view/2662/5147.

Barcellos, M. D. (2002). Processo decisório de compra de carne bovina na cidade de Porto Alegre (Dissertação de Mestrado). Universidade Federal do Rio Grande do Sul, Porto Alegre, Brasil. http://hdl.handle.net/10183/5188.

Behbahani, B. A., Noshad, M., \& Jooyandeh, H. (2020). Improving oxidative and microbial stability of beef using Shahri Balangu seed mucilage loaded with Cumin essential oil as a bioactive edible coating. Biocatalysis and Agricultural Biotechnology, 24, e101563. https://doi.org/10.1016/j.bcab.2020.101563.

Bermúdez-Oria, A., Rodríguez-Gutiérrez, G., Rubio-Senet, F., Fernández-Prior, A., \& Fernández-Bolaños, J. (2019). Effect of edible pectin-fish gelatin films containing the olive antioxidants hydroxytyrosol and 3,4-dihydroxyphenylglycol on beef meat during refrigerated storage. Meat Science, 148, 213-218. https://doi.org/10.1016/j.meatsci.2018.07.003.

Bhagath, Y. B., Manjula, K. (2019). Influence of composite edible coating systems on preservation of fresh meat cuts and products: a brief review on their trends and applications. Internation Food Research Journal, 26(2), 377-392. http://www.ifrj.upm.edu.my/26\%20(02)\%202019/(03).pdf.

Borges, C. B., Nunes, T. B. Neto, \& Cavalcante, J. M. M. (2020). Perfil do consumidor de carne bovina no município de Uruçuí-PI. PubVet - Medicina Veterinária e Zootecnia, 14(3), 1-9. http://www.pubvet.com.br/artigo/6827/perfil-do-consumidor-de-carne-bovina-no-municiacutepio-de-uruccediluiacute-pi.

Brandão, F. S., Ceolin, A. C., Canozzi, M. E. A., Révillion, J. P. P., Barcellos, J. O. J. (2012). Confiança e agregação de valor em carnes com indicação geográfica. Arquivo Brasileiro de Medicina Veterinária e Zootecnia, 64(2), 458-464. https://doi.org/10.1590/S0102-09352012000200028.

Brisola, M. V., \& Castro, A. M. G. (2005). Preferências do consumidor de carne bovina do Distrito Federal pelo ponto de compra e pelo produtor adquirido. Caderno de Pesquisas em Administração, 12(1), 81-99. http://www.spell.org.br/documentos/ver/26962/preferencias-do-consumidor-de-carne-bovina-dodistrito-federal-pelo-ponto-de-compra-e-pelo-produto-adquirido/i/pt-br.

Cardoso, G. P., Dutra, M. P., Fontes, P. R., Ramos, A. L. S., Gomide, L. A. M., \& Ramos, E. M. (2016). Selection of a chitosan gelatin-based edible coating for color preservation of beef in retail display. Meat Science, 114, 85-94. https://doi.org/10.1016/j.meatsci.2015.12.012.

Cazón, P., Velazquez, G., Ramírez, J. A., \& Vázquez, M. (2017). Polysaccharide-based films and coatings for food packaging: a review. Food Hydrocolloids, 68, 136-148, jul. 2017. https://doi.org/10.1016/j.foodhyd.2016.09.009.

Ceretta, S. B., Froemming, L. M. Geração Z: compreendendo os hábitos de consumo da geração emergente. (2011). Repositório da Universidade Potiguar, 3(2), 15-24. https://doi.org/10.21714/raunp.v3i2.70.

Cipriano, L. C., Siqueira, H. P. G., Messias, C. T., Lima, E. F., Medeiros, E. S., Hoppe, I. B. A. L., \& Siqueira, A. B. (2021). Perfil do consumidor de carne bovina no Estado de Roraima. Brazilian Journal of Development, 7(1), 1849-1864. https://doi.org/10.34117/bjdv7n1-126.

Coutinho, N. M., Silveira, M. R., Guimarães, J. T., Fernandes, L. M., Pimentel, T. C., Silva, M. C., Borges, F. O., Fernandes, F. A. N., Rodrigues, S., Freitas, M. Q., Esmerino, E. A., \& Cruz, A. G. (2021). Are consumers willing to pay for a product processed by emerging technologies? The case of chocolate milk drink processed by cold plasma. LWT - Food Science and Technology, 138, e110772. https://doi.org/10.1016/j.lwt.2020.110772.

Coray, T. Marketing para a Geração Z. https://www.sheerid.com/pt/blog/marketing-to-generation-z/.

Dias, L. D. B., Isernhagen, L., Brumatti, R. C., Faria, F. J. C., Franco, G. L., Kiefer, C., \& Ítavo, C. C. B. F. (2015). Estudo sobre o padrão de consumo da carne bovina na cidade de Campo Grande, MS, Brasil. Boletim de Indústria Animal, 72(2), 148-154. https://doi.org/10.17523/bia.v72n2p148

Eiras, C. E., Guerrero, A., Valero, M. V., Pardo, J. A., Ornaghi, M. G., Rivaroli, D. C., Sañudo, C., \& Prado, I. N. (2017). Effects of cottonseed hull levels in the diet and ageing time on visual and sensory meat acceptability from young bulls finished in feedlot. Animal, 11(3), 529-537. https://doi.org/10.1017/S1751731116001749.

Ellouze, M., \& Jeuge, S. (2014). Developmet of an edible protective coating for fresh meat: french consumer's perception. Les Cahiers de l'IFIP, 1(1), 69-74. https://ifip.asso.fr/.

Falguera, V., Quintero, J. P., Jiménez, A., Muñoz, J. A., \& Ibarz, A. (2011). Edible films and coatings: structures, active functions and trends in their use. Trends in Food Science \& Technology, 22(6), p. 292-303. https://doi.org/10.1016/j.tifs.2011.02.004.

Frabasile, D. (2016). Apenas em 3\% dos lares o homem toma as decisões de compra sozinho, mostra estudo. https://epocanegocios.globo.com/Marketing. 
Groot, E. (2021). Segmentos de preferências na aquisição da carne bovina. Revista de Economia e Sociologia Rural, 59(2), e213487. https://doi.org/10.1590/1806-9479.2021.213487

IBGE - Instituto Brasileiro de Geografia e Estatística. Boletim da educação PNAD contínua. 2019. https://ibge.gov.br.

Khedri, S., Sadeghi, E., Rouhi, M., Delshadian, Z., Mortazzavian, A. M., Guimarães, J. T., Fallah, M., \& Mohammadi, R. (2021). Bioactive edible films: development and characterization of gelatin edible films incorporated with casein phosphopeptides. LWT - Food Science and Technology, 138(4), e110649. https://doi.org/10.1016/j.lwt.2020.110649.

Kiarsi, Z., Hojjati, M., Behbahani, B. A., \& Noshad, M. (2020). In vitro antimicrobial effects of Myristica fragrans essential oil on foodborne pathogens and its influence on beef quality during refrigerated storage. Journal of Food Safety, 40(3), 1-14. https://doi.org/10.1111/jfs.12782.

Kirinus, J. K., Fruet, A. P. B., Klinger, A. C. K., Dörr, A. C., \& Nörnberg, J. L. (2013). Relação entre faixas de renda e o perfil dos consumidores de carne bovina da região sul do Brasil. Revista Monografias Ambientais, 12(12), 2776-2784. http://dx.doi.org/10.5902/2236130810424.

Lopes, M. A., Magalhães, D. R., Faria, P. B., Bruhn, F. R. P., Pereira, S. M., \& Demeu, F. A. (2017). Fatores associados a disposição de consumidores em adquirir carne bovina com certificação de origem na cidade do Rio de Janeiro, Brasil. Brazilian Journal of Veterinary Medicine, 39(2), 100-110. http://dx.doi.org/10.29374/2527-2179.bjvm027117.

Lorenzo, J. M., \& Pateiro, M. (2013). Influence of type of muscles on nutritional value of foal meat. Meat Science, 93(3), 630-638. https://doi.org/10.1016/j.meatsci.2012.11.007.

Magalhães, K. L., Andrade, A. P. C., Carvalho, J. D. G., \& Melo, M. L. B. (2021). Avaliação do perfil e dos hábitos de aquisição de consumidores de produtos cárneos em Fortaleza - CE. Research, Society and Developmente, 10(16), e162101623431. http://dx.doi.org/10.33448/rsd-v10i16.23431.

Maurício, R. A. (2020). Avaliação sensorial de carne bovina e ovina com revestimento comestível (Dissertação de Mestrado). Faculdade de Ciências Farmacêuticas, Universidade Estadual Paulista Júlio de Mesquita Filho, Araraquara, SP https://ainfo.cnptia.embrapa.br/digital/bitstream/item/215808/1/AvaliacaosSensorialCarne.pdf.

Mohamed, S. A. A., El-Sakhawy, M., \& El-Sakhawy, M. AM. (2020). Polysaccharides, protein and lipid -based natural edible films in food packaging: a review. Carbohydrate Polymers, 238(15), e116178. https://doi.org/10.1016/j.carbpol.2020.116178.

Moura, F.; Masquio, D. C. L. (2014). A influência da escolaridade na percepção sobre alimentos considerados saudáveis. Revista de Educação Popular, 13(1), 82-94. https://doi.org/10.14393/REP-v13n12014-art07.

Nascimento, J. D., Oliveira, D. M., Rocha, T. O. F., Rohod, R. V., Piazzon, C. J., Escobar, L. S. (2018). Perfil do consumidor de carnes do Alto Pantanal sulmato-grossense. Revista Acadêmica: Ciência Animal, 16, e16005. https://doi.org/10.7213/1981-4178.2018.16005.

Nikmaram, N., Budaraju, S., Barba, F. J., Lorenzo, J. M., Cox, R. B., Mallikarjuan, K., \& Roohinejad, S. (2018). Application of plant extracts to improve the shelf-life, nutritional and health-related properties of ready-to-eat meat products. Meat Science, 145, p. 245-255. https://doi.org/10.1016/j.meatsci.2018.06.031.

Ronteltap, A., Trijp, J. C. M., Renes, R. J., \& Frewer, L. J. (2007). Consumer acceptance of technology-based food innovations: lessons for the future of nutrigenomics. Appetite, 49(1), 1-17. https://doi.org/10.1016/j.appet.2007.02.002.

Sahraee, S., Milani, J. M., Regenstein, J. M, \& Kafil, H. S. (2019). Protection of foods against oxidative deterioration using edible films and coatings: a review. Food Bioscience, 32, e100451. https://doi.org/10.1016/j.fbio.2019.100451.

Schaly, L. M., Oliveira, M. C., Salviano, P. A. P., \& Abreu, J. M. (2010). Perfil do consumidor de produtos de origem animal de Rio Verde, GO. PubVet Medicina Veterinária e Zootecnia, 4(38), 962-967. https://www.pubvet.com.br/artigo/2549/perfil-do-consumidor-de-produtos-de-origem-animal-em-rio-verdegonbsp.

Schlindwein, M. M., \& Kassouf, A. L. (2006). Análise da influência de alguns fatores socioeconômicos e demográficos no consumo domiciliar de carnes no Brasil. Revista de Economia e Sociologia Rural, Rio de Janeiro, 44(3), 549-572. https://doi.org/10.1590/S0103-20032006000300009.

Souza, I. I. M. S., Lima, R. L. F. A., \& Silva, C. S. (2021). Revestimento bioativo à base de fécula de mandioca (Manihot esculenta) e beterraba acrescido de óleo de copaíba bálsamo (Copaifera officinalis) para conservação de carne bovina in natura. Research, Society and Development, 10(2), e15810212003. http://dx.doi.org/10.33448/rsd-v10i2.12003

Trejo-González, L., Rodríguez-Hernández, AI., López-Cuellar, M. R., Juárez-Martínez, VM., \& Chavarría-Hernández, N. (2018). Antimicrobial pectin-gellan films: effects on three foodborne pathogens in a meat medium, and selected physical mechanical properties. CyTA- Journal of Food, 16(1), 469-476. https://doi.org/10.1080/19476337.2017.1422278.

Tokin, E., Webb, T., Coveney, J., Meyer, S. B., \& Wilson, A. M. (2016). Consumer trust in the Australian food system - the everyday erosive impact of food labelling. Appetite, 103(1), 118-127. https://doi.org/10.1016/j.appet.2016.04.004.

Umaraw, P., Munekata, P. E. S., Verma, A. K., Barba, F. J., Singh, V. P., Kumar, P., \& Lorenzo, J. M. (2020). Edible films/coating with tailored properties for active packaging of meat, fish and derived products. Trends in Food Science \& Techonology, 98, 10-24. https://doi.org/10.1016/j.tifs.2020.01.032.

Utami, R., Kawiji, K. L. U., \& Nasution, M. I. A. (2017). Preservative effects of kaffir lime (Citrus hystrix DC) leaves oleoresin incorporation on cassava starch-based edible coatings for refrigerated fresh beef. International Food Research Journal, 24(4), 1464-1472. http://www.ifrj.upm.edu.my/24\%20(04)\%202017/(17).pdf.

Vital, A. C. P., Guerrero, A., Kempinski, E. M. B. C., Monteschio, J. O., Sary, C., Ramos, T. R., Campo, M. M., \& Prado, I. N. (2018). Consumer profile and acceptability of cooked beef steaks with edible and active coating containing oregano and rosemary essential oils. Meat Science, 143, 153-158. https://doi.org/10.1016/j.meatsci.2018.04.035.

Zhang, H., He, P., Kang, H., \& Li, X. (2018). Antioxidant and antimicrobial effects of edible coating based on chitosan and bamboo vinegar in ready to cook pork chops. LWT - Food Science and Technology, 93, 470-476. https://doi.org/10.1016/j.1wt.2018.04.005. 